\title{
Neutrino Nucleosynthesis in the outer layers of supernovae
}

\section{A. Sieverding*}

Institut für Kernphysik (Theoriezentrum), Technische Universität Darmstadt, Schlossgartenstraße 2, 64289 Darmstadt, Germany

E-mail: asiever@theorie.ikp.physik.tu-darmstadt.de

\section{Huther}

Institut für Kernphysik (Theoriezentrum), Technische Universität Darmstadt, Schlossgartenstraße 2, 64289 Darmstadt, Germany

\section{G. Martínez-Pinedo}

Institut für Kernphysik (Theoriezentrum), Technische Universität Darmstadt, Schlossgartenstraße 2, 64289 Darmstadt, Germany

\begin{abstract}
The influence of an extensive set of neutrino-induced reactions on the explosive nucleosynthesis in supernovae of solar metallicity stars with initial main sequence masses between 15 and $40 \mathrm{M}_{\odot}$ is studied. Cross-sections for the neutrino interactions are calculated for almost the whole nuclear chart including multi-particle evaporation are used.

${ }^{7} \mathrm{Li},{ }^{11} \mathrm{~B}$ and ${ }^{19} \mathrm{~F}$ are known to be produced in significant amounts by neutrino processes involving ${ }^{4} \mathrm{He},{ }^{12} \mathrm{C}$ and ${ }^{20} \mathrm{Ne}$. We focus on the production of the long-lived radioactive isotopes ${ }^{22} \mathrm{Na},{ }^{26} \mathrm{Al}$, ${ }^{44} \mathrm{Ti}$ and ${ }^{60} \mathrm{Fe}$. We find that the yields of ${ }^{26} \mathrm{Al}$ and ${ }^{22} \mathrm{Na}$ are increased on average by a factor of 3. There is only little effect on ${ }^{44} \mathrm{Ti}$ and ${ }^{60} \mathrm{Fe}$. Additionally, the production of ${ }^{92} \mathrm{Nb},{ }^{98} \mathrm{Tc},{ }^{138} \mathrm{La}$ and ${ }^{180} \mathrm{Ta}$ are studied. Significant contributions of the $v$-process to the production of these nuclei are found, in agreement with previous calculations and estimates.
\end{abstract}

XIII Nuclei in the Cosmos

7-11 July, 2014

Debrecen, Hungary

\footnotetext{
*Speaker.
} 


\section{Introduction}

Core-Collapse-Supernova explosions are the final stage of stellar nucleosynthesis and are crucial for the determination of the chemical composition of material that is ejected and enriches the interstellar medium, forming the basis for the next generation of stars. A major part of this nucleosynthesis happens as the shock front launched from the remnant of the stellar core passes through the layers of the star, increasing temperature and density and triggering nuclear processes. At the same time these layers are irradiated by neutrinos of all flavors, that are emitted as the remnant of the stellar core cools. The exact spectrum of the radiation is still uncertain, but neutrino energies are estimated to be of the order of $10 \mathrm{MeV}$. Via charged- and neutral-current interactions such neutrinos can lead to nuclear excitations beyond the particle separation threshold, affecting the chemical composition and density of free nucleons.

\section{Description of the Supernova and neutrino cross-sections}

We use an extensive set of neutrino-nucleus cross-sections to do nucleosynthesis network calculations for supernova explosions. The cross-sections have been calculated for both, charged- and neutral-current reactions, based on Random Phase Approximation (RPA) [1]. Spallation products in the case of states above particle separation threshold are computed based on statistical models [2]. The thermodynamic properties are parametrized following the description given by Woosley et al. [3]. Temperature and density evolution after the passage of the shock front are described as exponential decays with a dynamical timescale $\tau_{\mathrm{dyn}} \propto \rho_{0}^{-1 / 2}$, depending on the initial density $\rho_{0}$ at the considered radial zone. We use solar metallicity supernova progenitors calculated by A. Heger et al. [4], with main sequence masses ranging between 15 and $40 \mathrm{M}_{\odot}$. The stellar models have been evolved up to core-collapse, providing the initial conditions for the further evolution parametrized as stated above. For simplicity we assume, that the neutrinos follow a Fermi-Dirac distribution with zero chemical potential. The temperature characterizing the distribution is connected to the average energy via $\left\langle E_{v}\right\rangle=3.15 \times T_{v}$.

We assume a distribution with $T_{V}=4 \mathrm{MeV}$ for electron- and electron anti-neutrinos, corresponding to average energies of $\left\langle E_{V}\right\rangle=12.6 \mathrm{MeV}$. For the other neutrino flavors two cases are considered. $T_{v}=6 \mathrm{MeV}\left(\left\langle E_{v}\right\rangle=18.8 \mathrm{MeV}\right)$ and $T_{v}=8 \mathrm{MeV}\left(\left\langle E_{v}\right\rangle=25.1 \mathrm{MeV}\right)$ in order to compare the results to earlier calculations, even though simulations suggest much lower neutrino energies [5, 6].

\section{Impact on radioactive nuclei}

Observations of $\gamma$-rays allow direct access to the production of ${ }^{26} \mathrm{Al}$ in the galaxy [7]. Supernova explosions are assumed to give a significant contribution to the overall abundance of ${ }^{26} \mathrm{Al}$ [8]. Previous studies have already reported an enhancement of the production of ${ }^{26} \mathrm{Al}$ by $30 \%$ to $50 \%$ [9] due to neutrinos. This effect is largely due to neutral-current spallation reactions that increase the abundance of free protons, enhancing capture reactions on ${ }^{25} \mathrm{Mg}$ in the oxygen-neon shells. We observe an enhancement of the ${ }^{26} \mathrm{Al}$ production by neutrinos by an average factor of 2.9 for the set of progenitor stars considered (see figure $1(\mathrm{a})$ ). We are currently investigating the reasons for the 


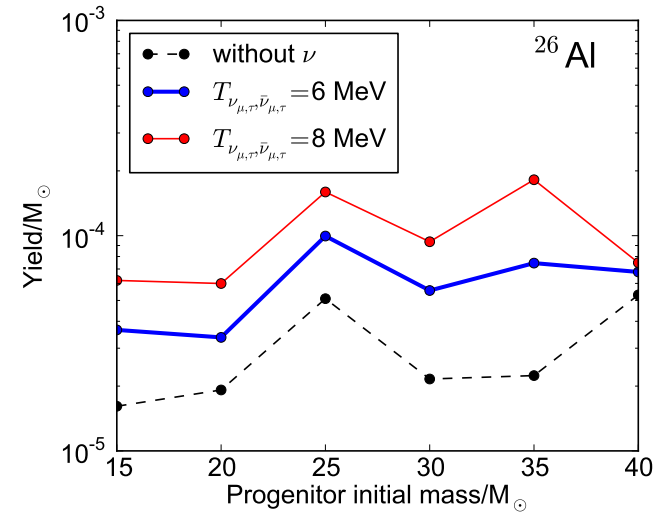

(a) ${ }^{26} \mathrm{Al}$ yield for a set of progenitor stars.

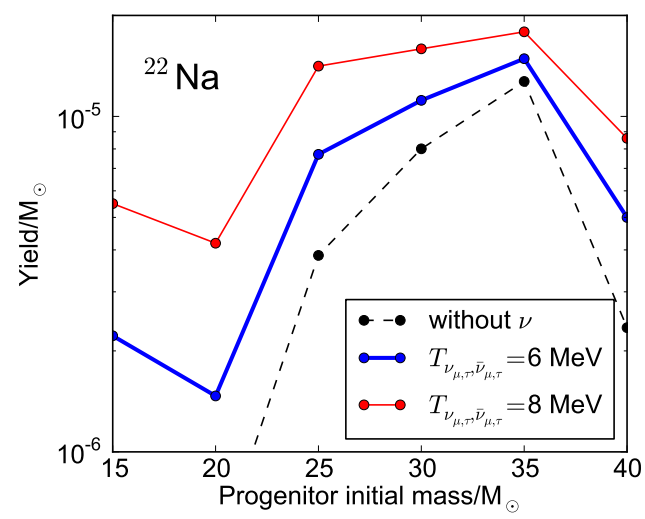

(b) ${ }^{22} \mathrm{Na}$ yield for a set of progenitor stars.

Figure 1: Shown are the results from calculations without neutrinos (dashed lines) and including neutrinos with different spectra. Electron neutrinos and anti-neutrinos are assumed have a distribution with $T_{v_{e}}=$ $T_{\bar{v}_{e}}=4 \mathrm{MeV}$.

differences compared to previous calculations. Apart from the effect of increased proton capture the charged-current reaction ${ }^{26} \mathrm{Mg}\left(v_{e}, \mathrm{e}^{-}\right)^{26} \mathrm{Al}$ contributes between $10 \%$ and $30 \%$ of the observed increase of the production. The neutral-current channels are dominant. This is reflected in the sensitivity to the temperature of the $\mu$-and $\tau$-(anti)-neutrino spectra.

The decay of ${ }^{22} \mathrm{Na}$ it is important for the description of supernova lightcurves [10]. In our calculations the production of ${ }^{22} \mathrm{Na}$ is increased on average by a factor of 3.1 (see figure 1(b)), mainly due to increased proton captures on ${ }^{22} \mathrm{Ne}$ and ${ }^{23} \mathrm{Na}\left(v_{e}, \mathrm{e}^{-} \mathrm{p}\right)^{22} \mathrm{Na}$. Further contributions are provided by the neutral current neutron evaporation on ${ }^{23} \mathrm{Na}$ and the charged-current reaction ${ }^{22} \mathrm{Ne}\left(v_{e}, \mathrm{e}^{-}\right)^{22} \mathrm{Na}$.

The characteristic $\gamma$-rays from the decay of ${ }^{44} \mathrm{Ti}$ and ${ }^{60} \mathrm{Fe}$ are also used as tracers for active nucleosynthesis sites $[8,11]$. The effect of neutrino interactions on the yields of ${ }^{44} \mathrm{Ti}$ and ${ }^{60} \mathrm{Fe}$ have been found to be at most $2 \%$ in the case of ${ }^{44} \mathrm{Ti}$ and even less for ${ }^{60} \mathrm{Fe}$.

\section{Impact on rare heavy nuclei}

${ }^{92} \mathrm{Nb},{ }^{98} \mathrm{Tc},{ }^{138} \mathrm{La}$ and ${ }^{180} \mathrm{Ta}$ are long-lived radioactive nuclei that have been observed in our solar system [12]. Their production, however, cannot be explained by neutron capture processes and the $\gamma$-process cannot account for the full abundance. Therefore, it has been suggested that those nuclei are produced mainly via charged-current neutrino reactions [13-15]. The processes contributing to the production of ${ }^{138} \mathrm{La}$ and ${ }^{180} \mathrm{Ta}$ have also been backed by experimental investigations [16].

Our calculations show, that significant amounts of those nuclei can be produced, when neutrino interactions are taken into account. ${ }^{92} \mathrm{Nb}$ and ${ }^{98} \mathrm{Tc}$ are found to be increased on average by factors of 50 (see figure 2). ${ }^{138} \mathrm{La}$ is enhanced by factors of more than 100 , giving an overproduction with 


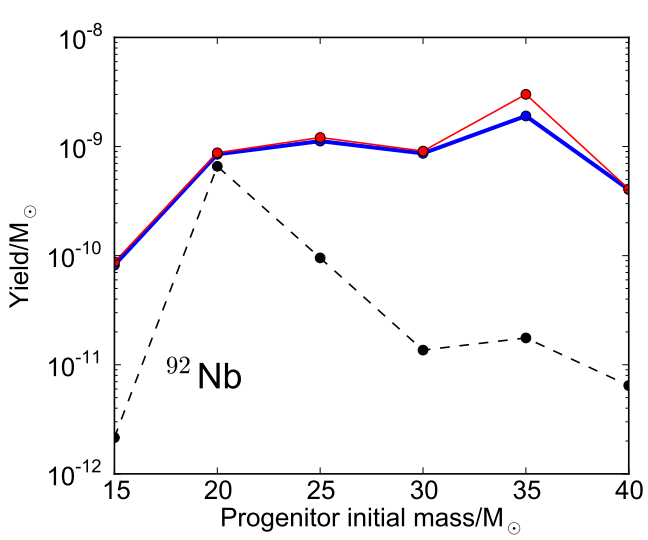

(a) ${ }^{92} \mathrm{Nb}$ yield for a set of progenitor stars.

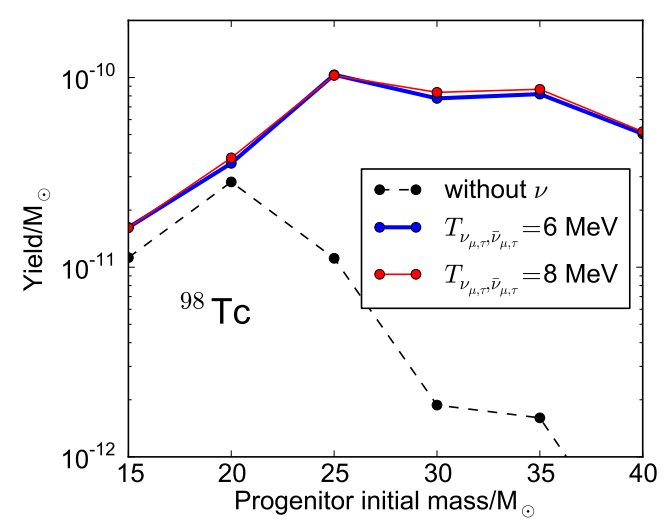

(b) ${ }^{98} \mathrm{Tc}$ yield for a set of progenitor stars.

Figure 2: Shown are the results from calculations without neutrinos (dashed lines) and including neutrinos with different spectra. Electron neutrinos and anti-neutrinos are assumed have a distribution with $T_{v_{e}}=$ $T_{\bar{v}_{e}}=4 \mathrm{MeV}$.

respect to solar isotopic ratios by a factor of 10 . The increases for ${ }^{92} \mathrm{Nb},{ }^{98} \mathrm{Tc}$ and ${ }^{138} \mathrm{La}$ are primarily due to electron neutrino absorption on the corresponding isobars. Therefore, their yield shows little sensitivity to the energies of the $\mu$ - and $\tau$-neutrinos and anti-neutrinos.

The production of ${ }^{180} \mathrm{Ta}$ has also significant contribution from neutron capture on ${ }^{179} \mathrm{Ta}$ in the context of the $\gamma$-process. With neutrinos increasing the density of free nucleons this contribution is also enhanced. In total, ${ }^{180} \mathrm{Ta}$ is increased by neutrinos by a factor of 12 . This result, however, does not yet take into account, that it is only the metastable $9^{-}$state at $77.1 \mathrm{MeV}$ that has a lifetime that is long enough to be detectable in the solar abundance pattern. According to an estimate given in [17] it is realistic to assume, that about $40 \%$ of the total production of ${ }^{180} \mathrm{Ta}$ remain in the metastable state. This assumption still gives an increase in the ${ }^{180} \mathrm{Ta}$ yield by a factor of almost 5 , due to neutrinos.

\section{Conclusions}

Table 1 summarizes the results and gives the production yield and factor relative to the yield in the case without neutrinos, averaged over the set of progenitor models. In addition to the nuclei discussed here, contributions have been found especially to the production of the light elements $\mathrm{Li}$ and $\mathrm{B}$, for which ${ }^{7} \mathrm{Li}$ and ${ }^{11} \mathrm{~B}$ are produced in agreement with previous studies in the full solar abundance. ${ }^{6} \mathrm{Li},{ }^{9} \mathrm{Be}$ and ${ }^{10} \mathrm{~B}$ however are underproduced with respect to the solar abundances. We see, that neutrino-induced reactions can influence the nucleosynthesis such that uncertainties with respect to supernova neutrinos also affect yield predictions. This can be especially important for the explanation of the observed abundances of ${ }^{26} \mathrm{Al}$.

Furthermore, the results confirm that neutrino nucleosynthesis can provide significant contributions to the production of the p-nuclei ${ }^{92} \mathrm{Nb},{ }^{98} \mathrm{Tc},{ }^{138} \mathrm{La}$ and ${ }^{180} \mathrm{Ta}$. 


\begin{tabular}{|c|l|r|}
\hline Nucleus & $\langle$ Yield $\rangle / \mathrm{M}_{\odot}$ & $\langle$ Factor $\rangle$ \\
\hline${ }^{7} \mathrm{Li}$ & $7.82 \times 10^{-7}$ & $4 \times 10^{13}$ \\
\hline${ }^{9} \mathrm{Be}$ & $7.83 \times 10^{-11}$ & $10^{11}$ \\
\hline${ }^{10} \mathrm{~B}$ & $1.79 \times 10^{-8}$ & $10^{11}$ \\
\hline${ }^{11} \mathrm{~B}$ & $3.25 \times 10^{-6}$ & $2 \times 10^{5}$ \\
\hline${ }^{19} \mathrm{~F}$ & $5.63 \times 10^{-5}$ & 1.17 \\
\hline${ }^{22} \mathrm{Na}$ & $7.07 \times 10^{-6}$ & 3.12 \\
\hline${ }^{26} \mathrm{Al}$ & $5.24 \times 10^{-5}$ & 2.94 \\
\hline${ }^{32} \mathrm{P}$ & $2.10 \times 10^{-4}$ & 19.07 \\
\hline
\end{tabular}

\begin{tabular}{|c|l|r|}
\hline Nucleus & $\langle$ Yield $\rangle / \mathrm{M}_{\odot}$ & $\langle$ Factor $\rangle$ \\
\hline${ }^{36} \mathrm{Cl}$ & $7.16 \times 10^{-5}$ & 22.20 \\
\hline${ }^{37} \mathrm{Ar}$ & $2.94 \times 10^{-4}$ & 1.74 \\
\hline${ }^{44} \mathrm{Ti}$ & $7.19 \times 10^{-5}$ & 0.98 \\
\hline${ }^{60} \mathrm{Fe}$ & $9.21 \times 10^{-5}$ & 0.99 \\
\hline${ }^{92} \mathrm{Nb}$ & $8.71 \times 10^{-10}$ & 47.60 \\
\hline${ }^{98} \mathrm{Tc}$ & $6.08 \times 10^{-11}$ & 50.14 \\
\hline${ }^{138} \mathrm{La}$ & $5.87 \times 10^{-10}$ & 123.22 \\
\hline${ }^{180} \mathrm{Ta}$ & $9.58 \times 10^{-12}$ & 11.86 \\
\hline
\end{tabular}

Table 1: Overview of the most significant effects of neutrinos on different nuclei. Given are the averages over the six progenitor models with equal weights. The average yield from the calculations including neutrinos with $T_{v_{\mu, \tau}}=T_{\bar{v}_{\mu, \tau}}=6 \mathrm{MeV}$ and $T_{v_{e}, \bar{v}_{e}}=4 \mathrm{MeV}$ and the factor is calculated relative to the results of the calculations without neutrinos. The values for ${ }^{180} \mathrm{Ta}$ are only from the $25 \mathrm{M}_{\odot}$ model.

\section{References}

[1] E. Kolbe, K. Langanke, G. Martínez-Pinedo, P. Vogel, Neutrino nucleus reactions and nuclear structure, Journal of Physics G Nuclear Physics 29 2569, [nucl-th/0311022 ].

[2] L. Huther, Nucleosynthesis in neutrino-driven winds, Ph.D. thesis, TU Darmstadt, Darmstadt, Germany (2013).

[3] S. E. Woosley, D. H. Hartmann, R. D. Hoffman, W. C. Haxton, The nu-process, ApJ. 356272.

[4] T. Rauscher, A. Heger, R. D. Hoffman, S. E. Woosley, Nucleosynthesis in Massive Stars with Improved Nuclear and Stellar Physics, ApJ. [astro-ph/0112478], URL http://2sn.org/nucleosynthesis/.

[5] G. Martínez-Pinedo, T. Fischer, L. Huther, Supernova neutrinos and nucleosynthesis, Journal of Physics G Nuclear Physics 41 (4) 044008, [ 1309.5477 ].

[6] H.-T. Janka, Explosion Mechanisms of Core-Collapse Supernovae, Annual Review of Nuclear and Particle Science 62 (1) 407.

[7] W. Wang, et al., Spectral and intensity variations of Galactic ${ }^{26}$ Al emission, A\&A496 713 , [0902.0211].

[8] M. Limongi, A. Chieffi, The Nucleosynthesis of ${ }^{26} A l$ and ${ }^{60} \mathrm{Fe}$ in Solar Metallicity Stars Extending in Mass from 11 to $120 \mathrm{M}_{\text {solar: }}$ The Hydrostatic and Explosive Contributions, ApJ. 647483 , [astro-ph/0604297].

[9] F. X. Timmes, S. E. Woosley, D. H. Hartmann, R. D. Hoffman, T. A. Weaver, F. Matteucci, 26Al and 60Fe from Supernova Explosions, ApJ. 449204.

[10] F.-K. Thielemann, M.-A. Hashimoto, K. Nomoto, Explosive nucleosynthesis in SN 1987A. II Composition, radioactivities, and the neutron star mass, ApJ. 349222.

[11] B. W. Grefenstette, et al., Asymmetries in core-collapse supernovae from maps of radioactive ${ }^{44}$ Ti in CassiopeiaA, Nature 506 339, [1403.4978 ].

[12] K. Lodders, Solar System Abundances and Condensation Temperatures of the Elements, ApJ. 591 1220. 
[13] S. Goriely, M. Arnould, I. Borzov, M. Rayet, The puzzle of the synthesis of the rare nuclide ${ }^{138} \mathrm{La}$, A\&A375 L35, [astro-ph/0107121].

[14] M.-K. Cheoun, et al., Neutrino induced reactions for $v$-process nucleosynthesis of ${ }^{92} \mathrm{Nb}$ and ${ }^{98} \mathrm{Tc}$, Phys. Rev. C85 (6) 065807, [1108.4229].

[15] A. Heger, E. Kolbe, W. C. Haxton, K. Langanke, G. Martínez-Pinedo, S. E. Woosley, Neutrino nucleosynthesis, Physics Letters B 606 258, [astro-ph/0307546].

[16] A. Byelikov, et al., Gamow-Teller Strength in the Exotic Odd-Odd Nuclei La138 and Ta180 and Its Relevance for Neutrino Nucleosynthesis, Physical Review Letters 98 (8) 082501.

[17] D. Belic, et al., Photoactivation of ${ }^{180} \mathrm{Ta}^{m}$ and Its Implications for the Nucleosynthesis of Nature's Rarest Naturally Occurring Isotope, Physical Review Letters 835242. 